\title{
A Review of the Animal and Human Trials of the Ad5-nCoV Vaccine Candidate
}

\author{
Cheng En $\mathrm{Xi}^{1}$, Jasrita Singh ${ }^{1}$ and Mark Larche ${ }^{1}$
}

${ }^{1}$ McMaster University, Hamilton, ON, Canada

DOI: https://doi.org/10.47611/jsr.v10i1.1159

\section{ABSTRACT}

Since the COVID-19 outbreak began, there has been an urgent need for a safe and effective vaccine to end this global pandemic. One such vaccine is the $\mathrm{Ad} 5-\mathrm{nCoV}$, developed by CanSino Biologics Inc. This review aims to examine all animal and human trials conducted for this vaccine candidate. Search terms such as "Ad5-nCoV", "recombinant adenovirus", "COVID-19", and "vaccine", were used in varying combinations in the PubMed database to find published trial reports. It was concluded that $\mathrm{Ad} 5-\mathrm{nCoV}$ can induce a strong immune response in mice and ferret models and offer them protection against the inoculation of SARS-CoV-2. It also has a strong safety profile in human and can induce an adequate immune response in terms of RBD-specific antibodies and $\mathrm{T}$ cell responses, while neutralizing antibody response and seroconversion was mediocre. The publish trial reports support the further testing of this vaccine candidate and it is preparing to enter phase 3 clinical trials.

\section{Introduction}

In March 2020, the World Health Organization (WHO) declared the COVID-19 outbreak to be a global pandemic. The virus responsible for the outbreak, SARS-CoV-2, was first detected in Wuhan, China, at the end of 2019. Since then, it has spread to every country in the world, resulting in more than 100 million confirmed cases and 2.4 million deaths worldwide.

On January 10, 2020, the genome of SARS-CoV-2 was sequenced and vaccine development began shortly after. One such vaccine in development is the Ad5-nCoV, by CanSino Biologics Inc. It is a replication-deficient type 5 human adenovirus vector (Ad5) encoding the gene for the SARS-CoV-2 spike protein. Adenovirus vectors, especially the ones isolated from primates like chimpanzees, have been previously used to develop vaccines for SARS6 and MERS - two zoonotic coronaviruses that are similar to SARS-CoV-2. Unlike other forms of COVID-19 vaccines, such as protein subunit or inactivated whole virus vaccines, adenovirus vector vaccines contain no pieces of the SARSCoV-2 virus. Instead, it contains DNA encoding the gene for its spike protein, enveloped in a modified adenovirus. The adenovirus will inject the DNA into human cells, where cells will use the DNA to produce the SARS-CoV-2 spike proteins using its own cellular machinery. This approach is theoretically safer than vaccines like whole virus vaccines5, as it carries no real pieces of SARS-CoV-2, making it impossible to get COVID-19 from the vaccine itself9. Adenovirus vaccines are also more stable than mRNA vaccines, like those developed by Pfizer-BioNTech and Moderna. Both the DNA and adenovirus used in this form of vaccine are tougher than the mRNA and lipid particles used by mRNA vaccines, allowing them to be stored at much higher temperatures than mRNA vaccines.

To assess the immunogenicity of a vaccine, metrics such as antibody response to the spike protein (S), specifically the receptor-binding domain (RBD), neutralizing antibody (NAb) response, and T cell response, are commonly used. Neutralizing antibodies differ from the RBD-specific antibodies in that they not only simply bind to a virus, but instead bind in a way that prevents infection. It can do so by blocking the virus' interaction with a receptor or prevent the uncoating of the viral genome. Only a small subset of antibodies are NAbs, but they are able to last 
longer to protect against future infections. One study conducted by Wajnberg et al. in New York City found that neutralizing antibody levels can persist for as long as five months after a SARS-CoV-2 infection.

Antibodies are proteins synthesized by B cells that work to inactivate foreign pathogens, like viruses, from the host body. The five classes of antibodies are $\operatorname{IgA}$, $\operatorname{IgD}$, $\operatorname{IgE}, \operatorname{IgG}$, and $\operatorname{IgM}$, each with a different biological property. An individual who develops antibodies to a pathogen has successfully undergone seroconversion, where antibodies in the blood become detectable. The spike protein of SARS-CoV-2, responsible for mediating viral entry into host cells and membrane fusion, is a key target for antibodies. The receptor-binding domain (RBD) of the spike protein has been found to bind strongly to human angiotensin-converting enzyme 2 (ACE2) receptors, which enables viral entry into human hosts. Thus, RBD has become an important target in vaccine development as blocking the RBD could inhibit their infection to host cells. To measure the antibody response induced by vaccines, the geometric mean titre (GMT) is commonly used as a measurement. It is the average concentrations of antibodies for a group of subjects involved in a trial. $\mathrm{T}$ cells are a type of leukocyte produced by the bone marrow and express a receptor that recognizes antigens from a variety of pathogens and tumours. T cells are divided into CD8+ and CD4+ T cells, the latter of which is further divided into subsets such as Th1, Th2, Th17, T-regulatory cells. CD8+ cells are cytotoxic and can induce apoptosis, while CD4+ cells work to activate the innate immune system, suppress immune reactions, and activate B cells for antibody production. $\mathrm{T}$ cells secrete cytokines such as interleukins, tumour necrosis factor (TNF) $\alpha$, and interferon (IFN) $\gamma$ to promote inflammation and macrophages, as well as activate other immune cells, making these cytokines a good indicator of $\mathrm{T}$ cell activity.

The objective of this review was to examine and summarize all published animal and clinical trial results of the Ad5-nCoV vaccine candidate as of August 20th, 2020. The summary will primarily focus on the methods, results, and discussions of each published trial report.

\section{Methods}

Key search terms of "Ad5-nCoV", "recombinant adenovirus", "SARS-CoV-2", "COVID-19", "vaccine", "human" were used in varying combinations in the PubMed database to look for all published clinical trial reports of the Ad5nCoV vaccine candidate. In addition, search terms of "animal", "Ad5-nCoV", "vaccine", "SARS-CoV-2", were also used in varying combinations in PubMed to find all published animal trial reports of the said vaccine candidate. Only original research articles published in the English language were reviewed for both searches. The first search found two published original research articles pertaining to human trials of the Ad5-nCoV vaccine candidate, with one on its phase 1 trial, and the other on its phase 2 trial. The second search found one published original research article regarding the animal trial of the said vaccine candidate. Each report was then thoroughly examined with a focus on the methods, results, and discussion sections for this review. All numerical data included in this review are significant, as they have a p-value $<0.05$, meaning there is a less than $5 \%$ that the values are due to chance.

\section{Results}

\section{Animal Model}

The animal model trial of Ad5-nCoV was published by Wu et al. in Nature Communications on August 14, 2020. The trial was conducted on both mice and ferret models and sought to determine the efficacy of administering the vaccine candidate through the intranasal route, in comparison to the regular intramuscular injection method. Metrics such as the production of antibodies, T cell responses, and the protective efficacy of Ad5-nCoV against the challenge of the SARS-CoV-2 virus in the animal subjects were examined.

The BALB/c mouse was selected to be the ideal mice model for this trial and was divided into groups of ten mice each. The mice were all female and between six and eight weeks old. Each group received one of the following 
doses: $5 \times 109$ virus particles (VP) (high dose), $5 \times 108 \mathrm{VP}$ (middle dose), or $5 \times 107 \mathrm{VP}$ (low dose) of Ad5-nCoV or $5 \times 109 \mathrm{VP}$ of the control vaccine (Ad5 vector). The administration of the viral particles was done via the intramuscular (IM) or the intranasal (IN) route. In addition, 10 weeks post-vaccination, 7 of 10 mice in every group were inoculated intranasally with the SARS-CoV-2 virus at a dose of 103.6 plaque-forming units (PFU). At 3 days post-inoculation (dpi), 4 of 7 mice in each group were sacrificed for viral load quantification in the lungs and turbinates through the use of quantitative polymerase chain reaction (qPCR) and plaque assay, with the other three being sacrificed for the same procedure at $5 \mathrm{dpi}$.

In addition to mice, ferrets were also used to assess the efficacy of Ad5-nCoV, as SARS-CoV-2 is able to efficiently infect the upper respiratory tract of ferrets 21,22 . The ferrets were divided into three groups ( $\mathrm{n}=18$ per group). Each group received one of the following treatments: IM injection $(5 \times 1010 \mathrm{VP})$, mucosal vaccination $(5 \times 1010 \mathrm{VP})$, and the control group. One ferret in the mucosal group also simultaneously received an IN delivery of Ad5-nCoV at the dose of the mucosal administration. At 4 weeks post-immunization, the ferrets were challenged with SARS-CoV-2 via the IN route at a dose of 105 PFU. Nasal washes were collected from the ferrets every two days for viral load analysis by qPCR and PFU assays.

Among the mice subjects, spike protein-specific (S-specific) IgG, anti-SARS-CoV-2-specific NAb, IgA, and other cellular immune responses were detected in each vaccination group through the collection of blood samples. ELISA IgG titres peaked at day 28 for the IM groups and slightly decreased afterwards, while the IN group mice remained at a steady peak from weeks 4 to 8 . The mice in the high-dose IN group had higher IgG titres than their IM counterparts at both weeks 6 and 8. No such difference was observed between the middle- or low-dose IM and IN groups at weeks 6 and 8. NAbs were measured using a SARS-CoV-2 specific microneutralization assay, which mixes serum antibodies at various dilutions with SARS-CoV-2 samples and exposes them to cells susceptible to infection in order to assess virus neutralization. The IN group reached the peak of NAb titre at week 6 and the IM group did so at week 8. The high-dose IN mice had significantly higher NAb titres than their IM counterparts from weeks 4 to 8 , while no difference was observed in the middle-dose group at weeks 6 and 8 , and none ever in the low-dose group. Both the middle-dose IM and IN groups showed significant induction of IFN $\gamma$ and IL-2 responses in splenic CD8+ T cells or CD4+ T cells at week 2, with a higher level in the IM group than in the IN group. Despite being infected with a mean viral load of $1.2 \times 104 \mathrm{PFU} / \mathrm{g}$ in the turbinates and 5.6 $\times 105 \mathrm{PFU} / \mathrm{g}$ in the lungs, no virus was detected using qPCR and PFU assays in either area of the IN mice at 3 and 5 dpi. The IM mice were infected with a mean viral load of $3.3 \times 106 \mathrm{PFU} / \mathrm{g}$, with no virus detected in the lungs. However, the virus was detected in the turbinates of some mice of the IM group by PFU assays and qPCR, with a significant reduction in the high and middle-dose groups compared to the control.

Among the ferret subjects, all vaccinated subjects produced S-specific IgG antibodies and NAb at week 4, with no difference between the two vaccination groups. The production of antibodies and NAb were not detected in the control subjects. qPCR and PFU assays detected no virus in the nose wash samples of the ferrets in the mucosal vaccination group from 2 to $8 \mathrm{dpi}$, while all control ferrets had infected samples. The virus was detected using PFU assay in the nose washes of 3/6,2/6, 0/6 and $0 / 6$ of the ferrets in the IM group at 2, 4, 6 and 8 dpi, respectively.

The data of this study demonstrated that a single dose of the Ad5-nCoV vaccine candidate can completely protect both the upper respiratory tract and lungs of mice, as well as producing a good immune response. In the ferret model, the vaccine candidate was also able to protect the respiratory tract of the subjects after 8 dpi. While the SARS-CoV-2 challenges administered to the animal subjects do not reflect realistic human exposure, it is of a much higher dose than one might encounter. Whether if the IM or mucosal vaccinations would adequately protect humans against infection remain to be tested in future human clinical trials.

\section{Phase 1 Clinical Trial}

The first human trial of the Ad5-nCoV vaccine candidate was published by Zhu et al. in The Lancet on June 13, 2020. It was a dose-escalation, single-centre, open-label, non-randomized, phase 1 trial, aiming to assess the safety, 
tolerability, and immunogenicity of this vaccine candidate. A total of 108 adults between the age of 18 and 60 were selected from Wuhan, China, with no individual having previously been infected by SARS-CoV-2, as confirmed by antibody rapid test kits, nucleic acid tests, and CT imaging to look for the presence of lung lesions. The participants were equally allocated to three dose groups ( $\mathrm{n}=36$ per group): one vial (low), two vials (middle), and three vials (high). Each vial contained $5 \times 1010 \mathrm{VP}$ per $0.5 \mathrm{~mL}$, and the vaccine candidate was administered as an IM injection. Due to the lack of placebo control, all participants stayed in a designated hotel for two weeks post-vaccination to eliminate live SARS-CoV-2 circulation and exposure as a confounding variable. Each participant self-reported adverse events and blood samples were taken to assess binding antibody responses against the RBD and spike glycoprotein with ELISA kits. NAb was measured using a live SARS-CoV-2 virus neutralization test. SARS-CoV-2 specific T cell responses were quantified with an IFN $\gamma$ enzyme-linked immunospot (ELISpot) assay, and CD4+ and CD8+ T-cell responses were also assessed according to the secretion of IFN $\gamma$, IL-2, and TNF $\alpha 10$.

Within the first seven days after the vaccination, at least one adverse event was reported in $83 \%$ (30/36) of the low and middle dose groups, and 75\% (27/36) of the high dose group. The detailed incidence rates of the most common adverse events are listed in Table 110.

Table 1. The incidence rate of local or systemic adverse events among the participants in each dose group ( $\mathrm{n}=36$ per group) who received the Ad5-nCoV vaccine candidate in phase 1 trial.

\begin{tabular}{|c|c|c|c|}
\hline & $\begin{array}{c}\text { Incidence rate in the low dose } \\
\text { group }\end{array}$ & $\begin{array}{c}\text { Incidence rate in the middle } \\
\text { dose group }\end{array}$ & $\begin{array}{c}\text { Incidence rate in the high dose } \\
\text { group }\end{array}$ \\
\hline Fatigue & $47 \%$ & $39 \%$ & $44 \%$ \\
\hline Fever & $42 \%$ & $42 \%$ & $56 \%$ \\
\hline Headache & $39 \%$ & $31 \%$ & $47 \%$ \\
\hline $\begin{array}{l}\text { Pain at in- } \\
\text { jection site }\end{array}$ & $47 \%$ & $56 \%$ & $58 \%$ \\
\hline Muscle pain & $19 \%$ & $8 \%$ & $22 \%$ \\
\hline
\end{tabular}

Across the treatment groups, no significant difference in the overall number of adverse events was observed, with most adverse reactions being mild or moderate. In total, nine participants, two each in the low and middle dose groups, and five in the high dose group, reported a case of severe fever with an axillary temperature greater than $38.5^{\circ} \mathrm{C}$. However, these reactions occurred within $24 \mathrm{~h}$ post-vaccination and lasted no more than $48 \mathrm{~h} 10$.

Antibody response to the RBD was observed in all dose groups from day 14, with the GMT of the binding antibodies being dose dependent. The GMTs were 1445.8, 806.0, and 615.8, in the high, middle, and low dose groups, respectively. More than $90 \%$ of the participants in all dose groups saw at least a four-fold increase in anti-RBD antibodies. NAb peaked on day 28 for all groups, and a NAb GMT of 34.0 was recorded in the high dose group, significantly higher than the 16.2 in the middle, and the 14.5 in the low dose group. On day $28,50 \%$ of the participants in the low and middle dose groups, and $75 \%$ in the high dose group, had at least a four-fold increase in NAb. T cell responses were significantly higher in the high dose group compared to that in the low dose group, but not significant when compared to the middle dose group. On days 14 and 28, IFN $\gamma$ was detected from CD4+ and CD8+ T cells across all dose groups, and on day 14, the TNF $\alpha$ expression from CD4+ T cells in the low dose group was significantly lower than the other two groups 10 .

To the knowledge of the team who conducted the study, this published report was the first one that covers an in-human clinical trial of a COVID-19 vaccine using adenovirus vectors. The vaccine candidate was found to be tolerated in all three dose groups among the healthy adult participants, with most adverse events being mild or moderate, and all were self-limiting. The higher reactogenicity of the high dose of $1.5 \times 1011 \mathrm{VP}$ was noted. The low dose $(5 \times 1010 \mathrm{VP})$ and middle dose $(1 \times 1011 \mathrm{VP})$ were selected to be further tested in the phase 2 clinical trial. The vaccine candidate was also able to induce humoral and $\mathrm{T}$ cell responses rapidly in most participants. Just a single dose of 
AD5-nCoV was able to elicit a four-fold increase in binding antibodies to RBD in 94-100\% of participants, and a four-fold increase to live SARS-CoV-2 in 50-75\% of participants 10 .

The limits of this study were the small sample size, lack of participants from the older population, the short follow-up period, and the lack of a randomized control. The main aim of this trial was to assess the safety, tolerability, and immunogenicity of the vaccine candidate in humans, and the trial found that Ad5-nCoV elicited mostly mild and moderate adverse events, while generating anti-RBD antibodies in more than $90 \%$ of the participants. These results show that the vaccine candidate is safe and tolerable in humans, as well as being able to generate antibody responses against SARS-CoV-2. The study also found that pre-existing immunity to Ad5 could slow down the immune responses to SARS-CoV-2, as well as lowering the peak of responses. This needs to be further studied in further trials 10.

\section{Phase 2 Clinical Trial}

The phase 2 clinical trial was also conducted by Zhu et al., and its results were published in The Lancet on August 15, 2020. This study was a placebo-controlled, randomized, double-blind trial, involving a total of 508 adult participants from Wuhan. It aims to determine an appropriate dosage of this vaccine candidate to be used for a phase 3 efficacy study. Exclusion criteria included previous SARS-CoV-2 infections, major chronic illnesses, and pregnant women. In total, 253 participants were given the $1 \times 1011$ VP dose (middle dose), 129 participants were given the $5 \times 1010 \mathrm{VP}$ (low dose), and 126 participants were given the placebo, with all doses being IM injections. All laboratory techniques (e.g., assays) for screening participants and assessing immune responses remain the same as the Phase 1 clinical trial. Like the phase 1 trial, participants also self-reported any adverse events.

Two weeks post-vaccination, 72\% (183/253) of the participants in the middle dose group and 74\% (96/129) of the participants in the low dose group reported adverse events, significantly higher than the 37\% (45/126) in the placebo group. The detailed incidence rates of the most common adverse events are listed in Table 2.

Table 2. The incidence rate of local or systemic adverse events among the participants in each dose group who received the Ad5-nCoV vaccine candidate in phase 2 trial.

\begin{tabular}{|c|c|c|c|}
\hline & $\begin{array}{c}\text { Incidence rate in the placebo } \\
\text { group }(\mathrm{n}=253)\end{array}$ & $\begin{array}{c}\text { Incidence rate in the low } \\
\text { dose group }(\mathrm{n}=129)\end{array}$ & $\begin{array}{c}\text { Incidence rate in the middle } \\
\text { dose group }(\mathrm{n}=126)\end{array}$ \\
\hline Fatigue & $17 \%$ & $42 \%$ & $34 \%$ \\
\hline Fever & $10 \%$ & $16 \%$ & $32 \%$ \\
\hline Headache & $13 \%$ & $28 \%$ & $57 \%$ \\
\hline $\begin{array}{c}\text { Pain at in- } \\
\text { jection site }\end{array}$ & $9 \%$ & $56 \%$ & $15 \%$ \\
\hline Muscle pain & $2 \%$ & $18 \%$ & $13 \%$ \\
\hline Joint pain & $3 \%$ & $10 \%$ & \\
\hline
\end{tabular}

Most adverse events were reported as either mild or moderate, with only $9 \%(24 / 253)$ of the participants in the middle dose group reporting severe adverse events, much higher than the other two groups.

From day 14 and onwards, RBD-specific antibodies were detected, with GMTs of 94.5 and 85.1 in the middle and low dose groups, respectively. This value peaked on day 28, with a GMT of 656.5 in the middle dose group and 571.0 in the low dose group. The participants in the control group showed no antibody increase from baseline. Both the middle and low dose groups induced significant NAb response to live SARS-CoV-2, with GMTs of 19.5 and 18.3, respectively. Seroconversion of NAb to live SARS-CoV-2 at day 28 occurred in 59\% (148/253) of the participants in the middle dose group, and 47\% (61/129) of those in the low dose group. Like the phase 1 study, pre-existing immunity to Ad5 resulted in lower RBD-specific antibody response and NAb response. Increasing age was found to be another independent negative factor on those two metrics, with those being 55 years or older having relatively lower antibody responses. Nonetheless, older participants in the two vaccine groups still showed higher antibody responses than those 
in the placebo group. On day 28, the vaccine candidate also induced significant S-specific IFN $\gamma$-ELISPot T cell responses in $90 \%$ (227/253) of the participants in the middle dose group, and 88\% (113/129) of those in the low dose group. No such responses were detected in the placebo group.

Compared to the phase 1 study, this study benefitted from a much larger sample size, as well as improvements like a placebo control, randomization, and double blinding. The study found that a single injection of Ad5-nCoV at $1 \times 1011$ VP or $5 \times 1010 \mathrm{VP}$ was able to induce comparable specific immune responses to the S-protein and specific $\mathrm{T}$ cell responses on day 28 . It also showed that the vaccine candidate has a good safety profile, as most adverse events were mild or moderate, and resolved within $48 \mathrm{~h}$. This is consistent with the phase 1 trial. Pre-existing anti-Ad5 immunity was found to be an obstacle for this vaccine candidate, as those with this form of immunity showed a relatively lower immune response. An additional dose might be able to overcome this.

The limitations of this study include the lack of representation of a global population (anti-Ad5 immunity varies by region), unavailability of participant data past day 28, and the lack of SARS-CoV-2 exposure to participants post-vaccination to assess the efficacy of this vaccine candidate. Given the results of this phase 2 trial, the researchers determined that further testing of Ad5-nCoV should be done with a dose of $5 \times 1010 \mathrm{VP}$, which will take place in a phase 3 efficacy trial. The limitations of this phase 2 trial design also emphasize the need for the phase 3 trial to be an international, multi-centre trial.

\section{Discussion}

The technology used by the Ad5-nCoV vaccine candidate has been previously used and tested in the development of a MERS7 vaccine, making the positive results of its trials relatively expected. In addition, other adenovirus vector vaccines being developed by other teams at the same time, notably the ChAdOx1 nCov-19 by the University of Oxford, have also seen positive results in their animal and human trials. Adenovirus vectors are the most commonly used vectors in gene therapies and are also the most common vector used for vaccines. There are at least 57 different types of adenovirus vectors (Ad1-Ad57) in humans, and most people have been infected with one or more types, with these infections being asymptomatic or mild in individuals with normal immune systems. Type 5 adenovirus, like the one used in Ad5-nCoV, is among the most common types of adenovirus. It is usually acquired during childhood and leads to upper respiratory tract infections. Most modified adenovirus vectors are Ad5s, which in the case of vaccines, are made replication-defective. This is done through the deletion of the E1A and E1B genes of Ad5.Replication-defective Ad5 vectors have been previously used in a tuberculosis vaccine trial, with promising results. The Ad5 vector used in that trial, named AdHu5Ag5A, was found to boost CD4+ and CD8+ T cell immunity in volunteers previously immunized with the Bacille Calmette-Guerin (BCG) vaccines.

From the human trials, it can be seen that $\mathrm{Ad} 5-\mathrm{nCoV}$ does indeed have a strong safety profile, with selflimiting adverse events. However, in its phase 1 trial, only $50 \%$ of the participants in the low and middle dose groups - the two doses that continued onto phase 2 trials, experienced a four-fold increase in NAb response in phase 1 trial10. The low proportion of NAb responses is further demonstrated in the phase 2 trial, as only $59 \%$ and $47 \%$ of the participants in the middle and low dose groups, respectively, had a seroconversion of NAb to live SARS-CoV-2 at day 2823. While other metrics, such as RBD-specific antibody response and $\mathrm{T}$ cell responses do seem adequate for this vaccine candidate in both human trials, the lower response in terms of NAb should be noted.

The design of the phase 2 study of this vaccine candidate is a major improvement from the phase 1 study. It should be expected that the phase 3 study of this vaccine candidate will make further improvement from the phase 2 design, notably including a larger and more ethnically and geographically diverse sample size by conducting the trial in multiple countries outside China. Another limitation is that only one trial was conducted for each stage (animal, phase 1 , and phase 2), thus, the vaccine candidate can benefit from several repeated trials of the same stage with different sample populations. However, given the urgency of COVID-19, it is unlikely that will be conducted, as even the phase 2 trial began before the completion of the data analysis of phase 1 trial23. This may be resolved in its phase 3 trials, as CanSino Biologics has announced that phase 3 trials will be conducted in several different countries, 
including Pakistan and Russia. However, it remains unclear as of now whether the trials in different countries will be published separately, in different original research articles. Since Ad5-nCoV has displayed a strong safety profile, its phase 3 studies should be focused on the effectiveness of the vaccine candidate on a global population, while paying close attention to the induced NAb responses and the effect of pre-existing anti-Ad5 immunity on immune responses.

\section{Conclusion}

The results from the animal trial of Ad5-nCoV show that the vaccine candidate is capable of producing a strong immune response in the mice and ferret subjects, as well as offer them protection from a high dose of IN SARS-CoV2 challenge. The phase 1 and 2 human trials showed that the vaccine is tolerable and has an adequate safety profile in humans, as well as inducing capable RBD-specific antibody response and T cell response, while the NAb response and seroconversion being less adequate. The data published in the phase 2 trial supports the continued assessment of Ad5-nCoV at a dose of $5 \times 10 \mathrm{VP}$ in the phase 3 clinical trial.

The results from the animal and clinical trials of Ad5-nCoV show that it is a viable vaccine candidate to be used against COVID-19 and its efficacy should be determined a in large-scale phase 3 trial. Future phase 3 trials should focus on assessing NAb response and seroconversion due to weaker data on that metric in phase 1 and 2 trials. Ad5-nCoV can also greatly benefit from an international, multi-centre trial, as all trials published as of August 20, 2020, have been conducted within China. A large-scale international trial can assess the tolerability and efficacy of the vaccine candidate on different ethnic populations, as well as gather more data on the impact of pre-existing antiAd5 immunity.

\section{References}

1. WHO Director-General's opening remarks at the media briefing on COVID-19 - 11 March 2020 [Internet]. Available from: https://www.who.int/dg/speeches/detail/who-director-general-s-opening-remarks-at-the-mediabriefing-on-covid-19---11-march-2020.

2. Li Q, Guan X, Wu P, Wang X, Zhou L, Tong Y, et al. Early transmission dynamics in Wuhan, China, of novel coronavirus-infected pneumonia. N Engl J Med. 2020; 382(13):1199-207. doi: 10.1056/NEJMoa2001316.

3. COVID-19 Map [Internet]. Johns Hopkins Coronavirus Resource Center. Available from: https://coronavirus.jhu.edu/map.html.

4. Jackson LA, Anderson EJ, Rouphael NG, Roberts PC, Makhene M, Coler RN, et al. An mRNA vaccine against SARS-CoV-2 — Preliminary Report. N Engl J Med. 2020;NEJMoa2022483. doi: 10.1056/NEJMoa2022483.

5. Wu S, Zhong G, Zhang J, Shuai L, Zhang Z, Wen Z, et al. A single dose of an adenovirus-vectored vaccine provides protection against SARS-CoV-2 challenge. Nat Commun. 2020;11(1):4081. doi: 10.1038/s41467-02017972-1.

6. Kobinger GP, Figueredo JM, Rowe T, Zhi Y, Gao G, Sanmiguel JC, et al. Adenovirus-based vaccine prevents pneumonia in ferrets challenged with the SARS coronavirus and stimulates robust immune responses in macaques. Vaccine. 2007;25(28):5220-31. doi: 10.1016/j.vaccine.2007.04.065.

7. Folegatti PM, Bittaye M, Flaxman A, Lopez FR, Bellamy D, Kupke A, et al. Safety and immunogenicity of a candidate Middle East respiratory syndrome coronavirus viral-vectored vaccine: a dose-escalation, open-label, 
non-randomised, uncontrolled, phase 1 trial. Lancet Infect Dis. 2020;20(7):816-26. doi: 10.1016/S14733099(20)30160-2.

8. Lai C-C, Shih T-P, Ko W-C, Tang H-J, Hsueh P-R. Severe acute respiratory syndrome coronavirus 2 (SARSCoV-2) and coronavirus disease-2019 (COVID-19): The epidemic and the challenges. Int J Antimicrob Agents. 2020;55(3):105924. doi: 10.1016/j.ijantimicag.2020.105924.

9. Corum J, Zimmer C. How the Johnson \& Johnson Vaccine Works. The New York Times [Internet]. Available from: https://www.nytimes.com/interactive/2020/health/johnson-johnson-covid-19-vaccine.html.

10. Zhu F-C, Li Y-H, Guan X-H, Hou L-H, Wang W-J, Li J-X, et al. Safety, tolerability, and immunogenicity of a recombinant adenovirus type-5 vectored COVID-19 vaccine: a dose-escalation, open-label, non-randomised, first-in-human trial. The Lancet. 2020;395(10240):1845-54. doi: 10.1016/S0140-6736(20)31208-3.

11. Payne S. Immunity and resistance to viruses. In: Viruses [Internet]. Elsevier; 2017. p. 61-71. Available from: https://linkinghub.elsevier.com/retrieve/pii/B9780128031094000064.

12. Wajnberg A, Amanat F, Firpo A, Altman DR, Bailey MJ, Mansour M, et al. Robust neutralizing antibodies to SARS-CoV-2 infection persist for months. Science. 2020;370(6521):1227-30. doi: 10.1126/science.abd7728.

13. B cells and antibodies - Molecular Biology of the Cell - NCBI Bookshelf [Internet]. Available from: https://www.ncbi.nlm.nih.gov/books/NBK26884/.

14. Definition of Seroconversion [Internet]. MedicineNet. Available from: https://www.medicinenet.com/seroconversion/definition.htm.

15. Chi X, Yan R, Zhang J, Zhang G, Zhang Y, Hao M, et al. A neutralizing human antibody binds to the N-terminal domain of the Spike protein of SARS-CoV-2. Science. 2020;369(6504):650-5. doi: 10.1126/science.abc6952.

16. Tai W, He L, Zhang X, Pu J, Voronin D, Jiang S, et al. Characterization of the receptor-binding domain (RBD) of 2019 novel coronavirus: implication for development of RBD protein as a viral attachment inhibitor and vaccine. Cell Mol Immunol. 2020;17(6):613-20. doi: 10.1038/s41423-020-0400-4.

17. Clinical_changes_IK_final.pdf [Internet]. Available from: https://www.who.int/biologicals/expert_committee/Clinical_changes_IK_final.pdf.

18. Kumar BV, Connors T, Farber DL. Human T cell development, localization, and function throughout life. Immunity. 2018;48(2):202-13. doi: 10.1016/j.immuni.2018.01.007.

19. Nilsson J, Gonçalves I, Edsfeldt A. Chronic inflammation and atherosclerosis. In: Early Vascular Aging (EVA) [Internet]. Elsevier; 2015. p. 157-67. Available from:

https://linkinghub.elsevier.com/retrieve/pii/B9780128013878000168.

20. Luckheeram RV, Zhou R, Verma AD, Xia B. CD4+T Cells: Differentiation and Functions. Clin Dev Immunol. 2012;2012. doi: 10.1155/2012/925135. 
21. Shi J, Wen Z, Zhong G, Yang H, Wang C, Huang B, et al. Susceptibility of ferrets, cats, dogs, and other domesticated animals to SARS-coronavirus 2. Science. 2020;368(6494):1016-20. doi: 10.1126/science.abb7015.

22. Kim Y-I, Kim S-G, Kim S-M, Kim E-H, Park S-J, Yu K-M, et al. Infection and rapid transmission of SARSCoV-2 in ferrets. Cell Host Microbe. 2020;27(5):704-709.e2. doi: 10.1016/j.chom.2020.03.023.

23. Zhu F-C, Guan X-H, Li Y-H, Huang J-Y, Jiang T, Hou L-H, et al. Immunogenicity and safety of a recombinant adenovirus type-5-vectored COVID-19 vaccine in healthy adults aged 18 years or older: a randomised, doubleblind, placebo-controlled, phase 2 trial. The Lancet. 2020;396(10249):479-88. doi: 10.1016/S0140-

6736(20)31605-6.

24. Doremalen N van, Lambe T, Spencer A, Belij-Rammerstorfer S, Purushotham JN, Port JR, et al. ChAdOx1 nCoV-19 vaccination prevents SARS-CoV-2 pneumonia in rhesus macaques. bioRxiv. 2020;2020.05.13.093195. doi: 10.1101/2020.05.13.093195.

25. Folegatti PM, Ewer KJ, Aley PK, Angus B, Becker S, Belij-Rammerstorfer S, et al. Safety and immunogenicity of the ChAdOx $1 \mathrm{nCoV}-19$ vaccine against SARS-CoV-2: a preliminary report of a phase $1 / 2$, single-blind, randomised controlled trial. The Lancet. 2020;396(10249):467-78. doi: 10.1016/S0140-6736(20)31604-4.

26. Wold WSM, Toth K. Adenovirus Vectors for Gene Therapy, Vaccination and Cancer Gene Therapy. Curr Gene Ther. 2013;13(6):421-33.

27. Smaill F, Jeyanathan M, Smieja M, Medina MF, Thanthrige-Don N, Zganiacz A, et al. A human type 5 adenovirus-based tuberculosis vaccine induces robust $\mathrm{T}$ cell responses in humans despite preexisting anti-adenovirus immunity. Sci Transl Med. 2013;5(205):205ra134. doi: 10.1126/scitranslmed.3006843.

28. CanSino Biologics [Internet]. Trial Site News. Available from: https://www.trialsitenews.com/category/cansino-biologics/ 